\title{
A Multiple Myeloma Patient Presenting with Multiple Hepatic Masses
}

\author{
Ajay Dhakal ${ }^{\mathrm{a}, \mathrm{c}}$, Abhinav Chandra $^{\mathrm{b}}$
}

\begin{abstract}
A 47-year-old male with Multiple Myeloma (MM) presented with a history of worsening jaundice and intermittent abdominal discomfort for one month. Physical examination was unremarkable except for the icterus and palpable, nontender liver extending $2 \mathrm{~cm}$ below the right costal margin. The liver function test was more consistent with an obstructive pathology. MRCP showed at least six hepatic masses- largest of which measured $16.4 \times 11.2 \mathrm{~cm}$, severe upper abdominal and retroperitoneal adenopathy and moderate to severe intrahepatic biliary duct dilatation due to extrinsic compression of Common Bile Duct $(\mathrm{CBD})$ by a $6.0 \times 5.7 \mathrm{~cm}$ porta-hepatis mass. A CAT-scan guided hepatic-mass-biopsy showed plasmacytoma. Endoscopic Retrograde Cholangiopancreatography with stent placement in CBD was done and the patient was discharged upon clinical improvement. Multiple Myeloma is a monoclonal, plasma-cell neoplasm that usually produces large amount of a specific immunoglobulin. Though Extramedullary plasmacytoma (EMP) is not uncommon feature of $\mathrm{MM}$, it is rarely considered as a specific part of the clinical picture of MM. Though the most common mechanism for development of EMP is local growth of the malignant tissue outside the bone, it can also be formed in various distant organs via hematogenous spread of the malignant plasma cells. Hepatic plasmacytoma is considered a rare entity and literature is limited to few case notes. Out of two distinct pathologic variants of hepatic plasmacytoma, infiltrative plasma-cell lesion are more common that macroscopic-nodular form. There are reports of nodular-hepatic plasma cell tumor being diagnosed incidentally or presenting with clinical features of hepatocellular injury and/or cholestasis. We have presented a rare case of hepatic plasmacytoma in MM with intrahepatic and porta-hepatis macroscopic nodules occurring simultaneously. This clinical picture of obstructive jaundice with multiple liver masses, a porta-hepatis mass and severe abdominal adenopathy usually generates differential diagnoses of primary liv-
\end{abstract}

Manuscript accepted for publication August 20, 2013

${ }^{a}$ Internal Medicine, Maimonides Medical Center, Brooklyn, NY, USA

${ }^{b}$ Hematology/Clinical Oncology, Maimonides Medical Center,

Brooklyn, NY, USA

${ }^{\mathrm{c} C o r r e s p o n d i n g ~ a u t h o r: ~ A j a y ~ D h a k a l, ~} 864$ 49th street Apt D5 Brooklyn

NY, USA. Email: adhakal@maimonidesmed.org

doi: http://dx.doi.org/10.4021/jmc1470w er tumor, metastases, gall bladder carcinoma, cholangiocarcinoma or abscesses. If a patient has pre-existing multiple myeloma, plasmacytoma should invariably be added to the differential diagnosis.

Keywords: Plasmacytoma; Multiple myeloma; Extramedullary plasmacytoma; Hepatic plasmacytoma

\section{Introduction}

Plasmacytoma of liver is an uncommon finding in Multiple Myeloma (MM). Macroscopic nodular form of hepatic plasmacytoma is even rarer. We present an interesting case of multiple nodular hepatic plasmacytomas with concurrent porta-hepatis plasmacytoma presenting as obstructive jaundice in a patient with MM.

\section{Case Report}

A 47-year-old male with MM, diagnosed 8 months ago, presented with a history of worsening jaundice and intermittent abdominal discomfort for 1 month. He completed a 28-day cycle of Bortezomib-Cyclophosphamide-Dexamethasone chemotherapy regimen 6 weeks back. The abdominal dis-

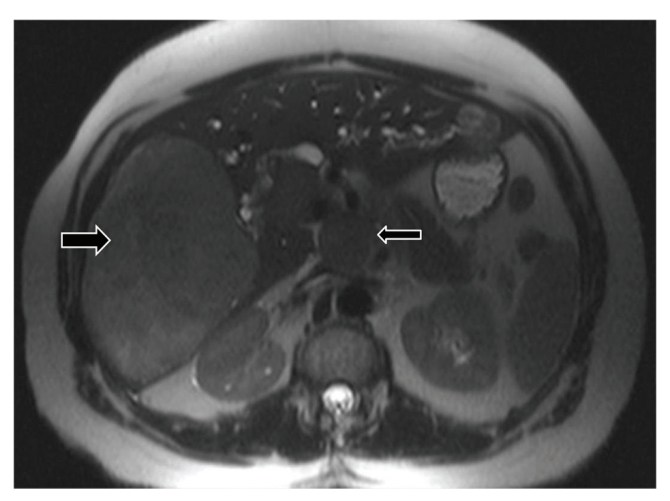

Figure 1. CT Scan of the abdomen showing intrahepatic (big arrow) and porta hepatic (small arrow) masses with intraabdominal and retro-peritoneal lymphadenopathy. 
comfort was not associated with nausea, vomiting, fever and he denied any history of hemolytic diseases or biliary stones. Physical examination was unremarkable except for the icterus and palpable, non-tender liver extending $2 \mathrm{~cm}$ below the right costal margin. The liver function test was more consistent with an obstructive pathology. MRCP showed at least 6 hepatic masses- largest of which measured $16.4 \times 11.2 \mathrm{~cm}$, severe upper abdominal and retroperitoneal adenopathy and moderate to severe intrahepatic biliary duct dilatation due to extrinsic compression of Common Bile Duct (CBD) by a $6.0 \times 5.7 \mathrm{~cm}$ porta-hepatis mass (Fig. 1). A CAT-scan guided hepatic-mass-biopsy confirmed plasmacytoma. Endoscopic Retrograde Cholangiopancreatography with stent placement in CBD was done and the patient was discharged upon clinical improvement. Patient was given outpatient appointments with his oncologist and a gastroenterologist in 2 weeks for further follow up.

\section{Discussion}

Multiple Myeloma (MM) is a monoclonal, plasma-cell neoplasm that usually produces large amount of a specific immunoglobulin. Bone marrow's plasma cell compartment constitutes most of the tumor burden of MM. But, atypical plasma-cells may also be found in the form of extramedullary plasmacytomas (EMP). Incidence of EMP is $7 \%$ to $18 \%$ at MM diagnosis and up to $20 \%$ at relapse [1]. It is argued that the incidence of EMP in MM is increasing as the treatment modality gets better leading to longer survival of the MM patients [1]. Common clinical manifestations of MM result from organ damage and tissue involvement causing hypercalcemia, renal insufficiency, anemia, and bone lesions- popularly known as CRAB symptoms. Despite the significant incidence of EMP in MM, it is rarely considered within the thought process during the management of MM. The most common mechanism for development of such plasma cell tumor is local growth of the malignant tissue outside the bone commonly seen in vertebrae, ribs, sternum and skull. It can also be formed in various distant organs via hematogenous spread of the malignant plasma cells typically involving reticulo-endothelial system and kidneys. Rarely, it can also be found in adrenals, urinary bladder, mesentery and liver [2]. Extramedullary progression of MM is associated with worse prognosis and presence of extramedullary involvement at any time during the disease course is associated with significantly shorter progression-free survival and overall survival $[1,3]$. Plasma cells of these EMP have more plasmablastic morphology, secrete more free light chains rather than intact immunoglobulins and thus considered more aggressive than plasma cells within the bone marrow. At diagnosis of MM, associated EMP can be sensitive to conventional agents, radiation therapy and high dose therapy. Single agent Thalidomide is found to produce poor response to EMP while
Lenalidomide is found to be more effective in such tumor. Literature is lacking on the proper management of EMP's and data about the preferred regimen are not based on direct comparison [1]. Considering the increase in the incidence of EMP in MM and its implication on the prognosis and the management of the disease, it should always be included as a part of the clinical picture of MM.

Hepatic plasmacytoma is considered a rare entity and literature is limited to few case notes. There are 2 distinct pathologic variants of hepatic plasmacytoma- infiltrative form and macroscopic nodular form; the former being much more common than the latter [4]. There are reports of nodular hepatic plasmacytoma being diagnosed incidentally or presenting with clinical features of hepatocellular injury and/ or cholestasis $[5,6]$. We have presented a rare case of hepatic plasmacytoma in MM with multiple intrahepatic nodules and simultaneous porta-hepatic macroscopic nodule. Up to our knowledge, such case of simultaneous porta hepatic and intra hepatic plasmacytomas creating a picture of obstructive jaundice in a patient with MM has never been reported in English language. This clinical picture of obstructive jaundice with multiple liver masses, a porta-hepatis mass and severe abdominal adenopathy usually generates differential diagnoses of primary liver tumor, metastases, gall bladder carcinoma, cholangiocarcinoma or abscesses. If a patient has pre-existing multiple myeloma, plasmacytoma should invariably be added to the differential diagnosis.

\section{Conclusion}

Extramedullary plasmacytoma (EMP) is rarely considered as a consistent part of the clinical picture of MM. Incidence of such tumors in MM can go up to $20 \%$ at relapse and is probably increasing with the increase in the survival of the MM patients in the era of novel agents. Furthermore, it imparts worse prognosis to $\mathrm{MM}$ and its specific management is lacking. Thus EMP can never be overlooked while managing a patient with MM. Hepatic plasmacytoma should definitely be in the differential diagnosis in a patient with MM who presents with multiple hepatic and/or porta hepatic masses and obstructive jaundice.

\section{Source of Support for the Study}

None.

\section{Prior Publication}

This manuscript has not been submitted or published anywhere else except for the abstract which was presented as a poster in both NY and National ACP poster competitions 2013. 


\section{Conflict of Interest}

None.

\section{Grant Support}

None.

\section{References}

1. Blade J, Fernandez de Larrea C, Rosinol L, Cibeira MT, Jimenez R, Powles R. Soft-tissue plasmacytomas in multiple myeloma: incidence, mechanisms of extramedullary spread, and treatment approach. J Clin Oncol. 2011;29(28):3805-3812.
2. Attwell A, Dee E, Russ P, Nash R, Shah R. Multiple myeloma involving the porta hepatis and peritoneum causing biliary obstruction and malignant ascites. Dig Dis Sci. 2005;50(6):1068-1071.

3. Aguado B, Inigo B, Sastre JL, Oriol A. Extramedullary plasmacytomas in the context of multiple myeloma. Adv Ther. 2011;28(Suppl 7):7-13.

4. Wu XN, Zhao XY, Jia JD. Nodular liver lesions involving multiple myeloma: a case report and literature review. World J Gastroenterol. 2009;15(8):1014-1017.

5. Musto P, Falcone A, Caturelli E, Squillante MM, Castelvetere M, Carotenuto M. Nodular lesions of the liver in multiple myeloma: a role for cytoadhesion molecules? Tumori. 1992;78(5):300-304.

6. Davis H, Caron GA, Mc KB. Multiple myeloma presenting clinically as obstructive jaundice: a case report. Postgrad Med J. 1959;35:668-670. 